\title{
Future directions for studying resilience of the oral ecosystem
}

\author{
Charifa Zemouri, ${ }^{1}$ Irina Dana Ofiteru² and Nicholas S. Jakubovics*3
}

\section{Key points}

There is an urgent need for a bette understanding of what constitutes a healthy and resilient oral ecosystem.
Longitudinal studies are essential for understanding resilience against oral disease.
Mathematical modelling approaches hold great promise for interpreting complex longitudinal data and identifying key biomarkers for healthdisease thresholds.

\begin{abstract}
The oral ecosystem is shaped by complex interactions between systemic health disease and the resident oral microbiota. Research in the last two decades has produced datasets describing the genetics and physiology of the host and the oral microbiome in health and disease. There are inter-individual differences in the ability to tolerate oral disease-promoting challenges. Identification of the key factors that drive a healthy and resilient oral ecosystem is urgently needed. So far, progress is being made towards replicating the host-microbiota interplay in vitro. Clinical studies may shed light on the mechanisms of oral health resilience. However, most clinical studies are cross-sectional and are insufficient for understanding resilience or for identifying biomarkers that correlate with the point of transition from oral health to dysbiosis. Mathematical and computational models, including artificial intelligence approaches, offer an opportunity to inform the design of clinical studies by identifying key biomarkers and interaction networks in complex datasets and predicting important parameters. This paper discusses some of the challenges and opportunities for understanding the biological basis of resilience of the oral ecosystem. It discusses the current status and challenges, and proposes a way forward to better understand resilience towards oral diseases.
\end{abstract}

\section{Introduction}

There has been intensive research on oral microorganisms for over one hundred years that has aimed to understand the disease state and how it comes about. ${ }^{1}$ However, before disease can be fully understood, it is necessary to have a clear definition of oral health, in terms of both the clinical signs and symptoms and the profile of key biomarkers that correlate with health. The oral cavity is a complex ecosystem and potential biomarkers include host immunological and biochemical mediators as well as the microbiota residing on different surfaces in the mouth. ${ }^{2,3}$ The large-scale identification of key factors that

'Department of Preventive Dentistry, Academic Centre for Dentistry Amsterdam, University of Amsterdam and Vrije

Universiteit Amsterdam, Amsterdam, The Netherlands:

${ }^{2} S$ chool of Engineering, Faculty of Science, Agriculture

and Engineering, Newcastle University, Newcastle upon

Tyne, UK; ${ }^{3}$ School of Dental Sciences and Biosciences

Institute, Faculty of Medical Sciences, Newcastle University,

Newcastle upon Tyne, UK.

${ }^{*}$ Correspondence to: Nicholas Jakubovics

Email address: nick.jakubovics@ncl.ac.uk

Refereed Paper.

Accepted 27 July 2020

https://doi.org/10.1038/s41415-020-2407-8 contribute to the maintenance of oral health requires holistic top-down approaches that can simultaneously monitor multiple factors within the complex environment of the human oral cavity. For example, the analysis of biochemical, metabolomic and microbiome constituents in saliva of 268 healthy adults has shown that multiple ecological states or 'ecotypes' are found in oral health. ${ }^{4}$ It is not yet known whether any of these ecotypes correlate with increased predisposition to oral disease. In addition, it is not clear how ecotypes change with the normal ageing process. There is evidence that both the host immune response and the oral microbiome change through the life course. ${ }^{5,6,7}$ Therefore, it is likely that additional ecotypes will be identified in individuals from different age groups. Although a great deal of research has been performed to identify individual species that correlate with oral health or disease, it is likely that the overall functions encoded and/ or expressed within the microbiome may be more important for determining health or disease status than the taxa present. It has been proposed that there may be a core set of functions required for a health-associated oral microbiome, just as a core set of human genes is needed for health. ${ }^{7,8}$

It is now well established that dental plaquerelated diseases such as dental caries and periodontitis are associated with shifts in the oral microbiome at the site of disease. ${ }^{1}$ This basic concept dates back to the 'Ecological Plaque Hypothesis' that was proposed by Marsh et al. in the 1990s to rationalise previous conflicting ideas that either (i) certain species (pathogens) are responsible for dental plaque-mediated diseases, or (ii) it is the amount of dental plaque rather than the species present that is important for driving disease. ${ }^{9,10,11}$ According to the Ecological Plaque Hypothesis, the development of disease involves an initial disturbance which is then amplified by a selection for microbial species that contribute to the disease process. In the case of dental caries, the process is driven by excess amount or frequency of sugars intake. This selects initially for moderately acidogenic genera such as Streptococcus or Actinomyces, which then further contribute to acid production in the local environment of dental plaque. ${ }^{12}$ Eventually, the population becomes enriched in more acidogenic species 
such as Streptococcus mutans (S. mutans) or Scardovia wiggsiae. ${ }^{13,14}$ S. mutans also produces insoluble glucans from sucrose that create pockets of low $\mathrm{pH}$ close to the tooth surface. ${ }^{15,16}$ In periodontitis, the interplay between bacteria and the host is critical for disease. The accumulation of dental plaque at the gingival margin is considered the primary trigger for the initiation of inflammation in most cases. ${ }^{9}$ The transition from gingivitis to periodontitis is associated with a shift in the population that particularly enriches gram-negative anaerobic proteolytic species including Socransky's 'red complex' Porphyromonas gingivalis (P. gingivalis), Tannerella forsythia and Treponema denticola, as well as grampositive anaerobes such as Filifactor alocis and a variety of other species. ${ }^{1,17,18}$ Using a mouse model of periodontitis, Hajishengallis et al. demonstrated that $P$. gingivalis can induce strong pathogenic host responses even when present at very low abundance in the oral microbiome. ${ }^{19}$ They designated this species a 'keystone pathogen' on the basis that its impact on disease is disproportionate to its abundance in the microbiome. ${ }^{20}$ They proposed a model whereby the commensal microbiota includes species that are incompatible with disease and those that can potentially promote disease in the presence of risk factors through interactions with keystone pathogens. These keystone pathogens trigger changes in the host (inflammation, in the case of subgingival biofilms), which then select for more pathogenic species termed pathobionts. Overall, this results in microbial dysbiosis and destructive host responses. ${ }^{20}$

While these models present a broad view of the distinction between oral health and disease, they are currently focused on explaining disease in terms of the microorganisms present rather than the functions that these species encode. In addition, current models do not yet account for underlying inter-individual differences in the capacity to tolerate cariogenic or periodontal disease drivers. ${ }^{21}$ The risk of oral diseases changes dramatically through the life course. Whereas dental caries is prevalent even among young age groups, the prevalence of severe periodontitis increases with age and appears to be higher in males than females. ${ }^{22}$ In each case, certain forms of disease are associated with particular life stages. For example, early childhood caries is an aggressive form of disease that affects the primary dentition whereas root caries occurs later in life. ${ }^{23,24}$ Similarly, forms of grade C periodontitis (formerly 'localised aggressive periodontitis') tend to onset around the time of puberty, between ages $11-13 .{ }^{25}$ Risk factors for caries and periodontitis are complex and it is not entirely clear how age impacts hostmicrobiome interactions. In the case of early childhood caries, enamel defects and modifiable risk factors such as sugar intake and low fluoride exposure influence disease development early in life. ${ }^{26}$ By contrast, ageing of the immune system ('immunosenescence') combined with a low-grade inflammation that develops during ageing ('inflammaging') are thought to impact the outcomes of the periodontitis challenge from the subgingival microbiota and potentially contribute to the increase in disease seen in older people. ${ }^{7,27}$ However, the limited does not directly change the oral microbiome to a great extent. ${ }^{27}$ Similarly, the oral microbiome is relatively stable over the short term in the absence of gross perturbations. ${ }^{28}$ It is thought that the microbiome is dynamic, and that stability is maintained by intrinsic factors that counter the changes and continually restore the system. ${ }^{29}$ Minor challenges can be absorbed within the natural dynamics of the system. The capacity to resist change will be dependent on the individual. If the perturbation overcomes this natural resistance, the system will depart evidence available to date indicates that ageing

from its natural homeostasis. The challenge for the individual is then to restore health rather than allowing a more extensive shift to dysbiosis or disease. The natural ability to revert to the original state or an alternative health-associated stable state is termed 'resilience' (Fig. 1).

In an excellent recent review, Rosier et al. have discussed some of the mechanisms that may determine resilience in the oral microbiome. ${ }^{30}$ These include mechanisms intrinsic to the microbiota (such as bacteriophage and the production of antimicrobials including bacteriocins and hydrogen peroxide that mediate inter-microbial competition), as well as host immunity and environmental modulators, such as nutrition and growth factors. In vitro and animal models have been developed to study the effects of these factors on preventing or reversing microbial dysbiosis and/or oral disease. ${ }^{31,32}$ However, these models have a limited capacity to characterise those components of the system that become important or increase in importance only when they interact with one another. In addition, animal models will never fully replicate all factors of human biology, even though advancements have been made towards developing models with 'humanised' versions of both immune system components and the

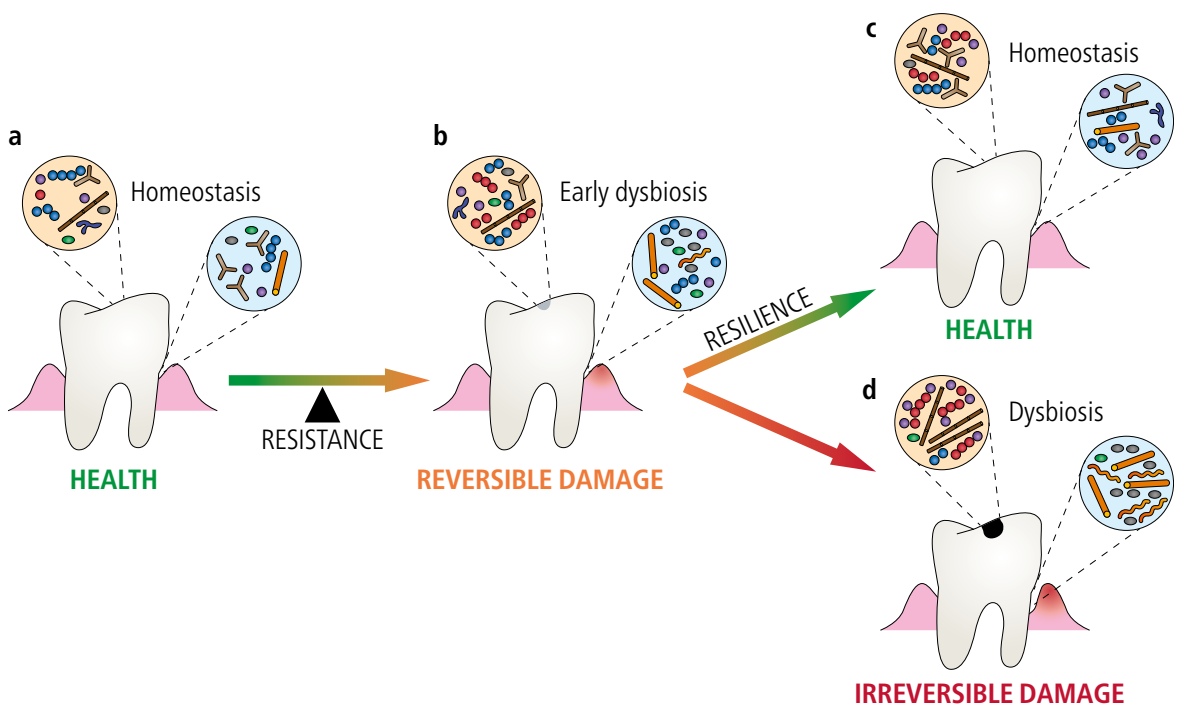

Fig. 1 Resistance and resilience of the oral ecosystem. a) In health, the supragingival (yellow) and subgingival (blue) dental plaque microbiomes are in a state of homeostasis. b) Each individual has a capacity to resist pathogenic changes that lead to early signs of dental caries (small grey area indicating early lesion) or gingivitis (reddening of the gingival tissue). Changes are associated with shifts in the microbiome. c) The ability to reverse the early signs of dental caries or gingivitis and restore oral health is termed resilience. This is accompanied by a restoration of the dental plaque microbiome. d) If early changes are not reversed and there is continued exposure to disease-promoting factors, irreversible damage ensues in the form of more significant carious lesions (black area on the tooth) or periodontitis (indicated as reddening/inflammation and the presence of a periodontal pocket). The microbiome associated with disease is in a state of dysbiosis 
microbiome. ${ }^{33,34}$ Therefore, there is still a need to conduct clinical studies on humans to gain a holistic understanding of the key features of the oral ecosystem that contribute to oral health. Resistance and resilience are temporal concepts and cannot be fully understood with cross-sectional studies. However, with the exception of the well-characterised experimental gingivitis model, ${ }^{35}$ relatively few studies have investigated longitudinal changes in the oral microbiome or in host inflammatory mediators through the course of cariogenic or gingivitis/periodontitis challenges. As a result, there are currently no strong biomarkers that can classify different levels of resistance or resilience, or that can explain the mechanistic basis of these differences.

\section{Evidence from longitudinal studies}

While biomarkers for resistance and resilience are urgently needed, interpreting the function of these markers is often not straightforward. Although there is a desire to identify host and microbiological changes that occur at the site of initiation of carious or periodontal lesions, this is almost impossible to achieve practically at present as it would require sampling from a very large number of sites in the mouths of many volunteers over an extended time period. Therefore, most longitudinal studies on the microbiology of periodontitis have focused on sites where disease is already present and have assessed responses to treatment rather than disease development. ${ }^{36,37}$

As noted by Rosier et al., ${ }^{30}$ the impact of microorganisms on other aspects of the innate immune system such as interleukin (IL)-8 can also be interpreted differently depending on whether species are associated with health and disease. Thus, both P. gingivalis and Streptococcus salivarius (S. salivarius) suppress IL-8 secretion. In the case of the keystone pathogen P. gingivalis, this is thought to aid virulence, ${ }^{38}$ whereas inhibition of IL-8 secretion by the oral commensal S. salivarius is considered to prevent unnecessary and destructive inflammation and contribute to homeostasis. ${ }^{39}$ However, P. gingivalis is also present at low levels in periodontal health and, in these circumstances, suppression of IL-8 production may help to maintain homeostasis. There is also evidence that the role of certain microorganisms in the transition from health to disease may be specific for one certain population and not others. ${ }^{40}$ These complexities make it difficult to find a robust biomarker that can identify the tipping point between health and irreversible disease.

Early longitudinal studies investigating the microbiology of dental caries relied on microbiological culture and were limited in their ability to sample the microbiome holistically. ${ }^{41,42,43}$ More recently, a number of longitudinal studies of dental caries have employed culture-independent microbiome analysis methods. ${ }^{44,45,46}$ Due to limitations with cost and sensitivity of the methods, these studies have so far used pooled dental plaque rather than plaque isolated from specific sites of caries development. It appears that shifts in the population in supragingival dental plaque precede the development of dental caries. ${ }^{46}$

The early stages of periodontal disease can be modelled experimentally in humans using the 'experimental gingivitis' approach that was pioneered by Löe et al. in the 1960s. ${ }^{47}$ We have recently reviewed the evidence for resistance and resilience to experimental gingivitis. ${ }^{35}$ Fluctuations of immunological, biochemical and microbiological biomarkers were identified throughout different phases and challenges to the oral cavity. In some cases, trends in biomarkers appeared to correlate with resilience. For example, lactoferrin was reported to increase in concentration during experimental gingivitis and return to baseline upon disease resolution. However, in general, few of the studies covered by the review had investigated the recovery phase following gingivitis and therefore it remains unclear which factors contribute to resilience.

\section{Mathematical opportunities to model challenges to an ecosystem}

Experimental in vivo studies face numerous challenges including the availability of participants, ethical regulations, cost, extended time to study the onset of a disease or clinical outcomes, and the fact they are limited in the range of conditions they can screen. Moreover, it is often difficult or impossible to identify mechanisms directly in human subjects. ${ }^{48}$ One promising approach to study challenges in the oral ecosystem is through mathematical modelling. ${ }^{49}$ Models have long been used in microbial ecology. ${ }^{50}$ A model can simulate any feasible hypothetical oral ecosystem or scenario to identify which parameters (biomarkers) have the strongest influence on an outcome. For example, a model can include hypothetical nutritional regimes to study perturbing factors, within any timeframe or exposure time, without having to burden study participants (for example, a sustained high/frequent sugar intake). The outcome of the simulations can then be used to inform targeted in vivo experiments.

Pioneering work on mathematical modelling in dentistry was developed by Dibbin and Reece. ${ }^{51}$ In the decades to follow, models were used to study disease development in caries research ${ }^{52,53,54}$ and progression of periodontitis. ${ }^{55,56}$ These examples cover a variety of model types and the choice of model should match the question asked. ${ }^{49,57}$ The oral microbiome is a complex ecosystem of dynamic multi-scalar, spatio-temporal interactions among organisms as well as between organisms and the environment. For questions that are not dependent on the spatial arrangement between species in dental plaque, a continuum model can be used. ${ }^{52,58}$ In Ilie et al. ${ }^{52}$ four microbial groups were selected as the main plaque components (aciduric Streptococcus, non-aciduric Streptococcus, Actinomyces and Veillonella). The study focused on the influence of different factors on the $\mathrm{pH}$ change in time and tooth demineralisation. In the absence of Veillonella, the $\mathrm{pH}$ values were estimated to decrease to $\mathrm{pH}$ 3. The $\mathrm{pH}$ variation was better explained by microbial activity rather than the buffering effect of the phosphate released by tooth demineralisation. Such output indicates that the presence of Veillonella, or other lactic acid-consuming bacteria, is an important indicator for a resilient or a healthy ecosystem. However, care should be taken in interpreting the data since, experimentally, Veillonella is often observed to be increased in caries, and may support the growth of acidogenic species such as $S$. mutans. This emphasises the need for validation in laboratory or clinical studies, as well as for understanding the mechanisms involved.

A continuous model cannot represent the competition for space within the plaque, the $\mathrm{pH}$ gradient or the localised effect of the dental plaque on the tooth. Agent-based models are better suited for this type of problem, though they have the disadvantage of increasing complexity and computational requirements..$^{54,59,60}$ An agentbased/cellular automata model was used by Papantonoupoulos et al. ${ }^{55,56}$ to simulate the progression of periodontitis disease and its dynamic characteristics. Their results suggest that periodontitis is a nonlinear, chaotic dynamical process in which the host immune response controls its progression rate. One 


\section{Fig. 2 Research loop for mathematical modelling leading to study setup}

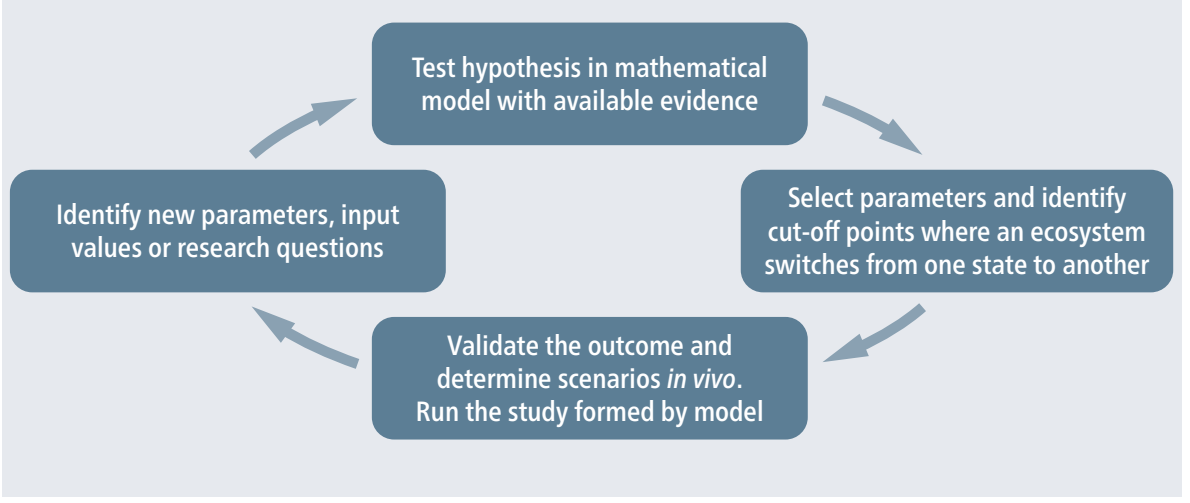

of the modelled findings was that, when host immune response increases, periodontitis progression rate decreases. The model was validated by using clinical and immunological data, and the data fit two distinct clinical manifestations that were differentiated at the time as aggressive or chronic periodontitis.

Continuous models and agent-based models are based on first principles. An alternative approach is the 'black box' models, such as artificial neural networks (ANNs), which use existing data as training sets to develop patterns that can predict outcomes. For example, ANNs have been used to discriminate between two types of periodontitis, ${ }^{61}$ using the patient's immune response profile. More recent advancements in artificial intelligencebased approaches ${ }^{62,63}$ need significantly more data and depend on coordinated international collaboration for the development of a global database repository. It would be interesting to map these approaches to the new periodontal classification scheme ${ }^{64}$ to determine whether they are able to distinguish between stages or grades of disease.

Based on these examples, we propose that a model can also be used to estimate cut-off points or critical values where the oral ecosystem will shift from a homeostatic state (during perturbing challenges) to a dysbiotic state. The advantages of modelling will allow the creation of a research loop to define research objectives and outcome variables (see Figure 2). First, a hypothesis can be tested in a mathematical model with available evidence. The parameters can be selected to identify cut-off points where an ecosystem switches from one state to another. The outcomes can be validated in vivo which methodology is informed by the model. The in vivo research outcomes then provide new evidence and parameters used as input values or to generate new research questions to be modelled. Notwithstanding the limitations of any model (for example, inherent simplifying assumptions), exploring changes of the ecosystem in a mathematical model is an inexpensive way to test hypotheses and to identify future research aspects to confirm the modelled outcome.

\section{Conclusion}

While many studies have investigated microbial and host factors that are associated with dental caries or periodontal disease, we are still a long way off understanding human health. In particular, the capacity of individuals to resist or recover from cariogenic or periodontal disease stimuli is not yet known. There is an urgent need for additional longitudinal studies in this area. Mathematical modelling has great potential to guide these studies and to establish testable hypotheses. However, investigations will need to include multiple measurements of host and microbial factors to provide sufficient information to start understanding the dynamics of changes in the microbiome in response to disease-inducing stimuli.

\section{Acknowledgements}

The authors would like to thank the Department of Preventive Dentistry and Department of Periodontology at ACTA for their insights and feedback on the data and interpretation. We would like to acknowledge the following specialists for their insights on this paper: Bruno Loos, Bryan Hanley, Colette Shortt, Egija Zaura, Lisa Rizzetto, Wim Crielaard, Betinna Schelkle and Michela Miani.

\section{Conflict of interest}

This study has been funded partly by the Foundation of Oral Biology (Stichting Orale Biologie), The

Netherlands. This work was conducted by an expert group from the Functional Foods Task Force of the European branch of the International Life Sciences Institute (ILSI Europe). Industry members of this task force are listed on the ILSI Europe website at http:// ilsi.eu/task-forces/nutrition/functional-foods/. Experts are not paid for the time spent on this work; however, the non-industry members within the expert group received a small compensatory sum (honouraria) and travel support from the Functional Foods Task Force to attend meetings to discuss the review. ILSI Europe was not involved in the design of the study, the collection, analysis and interpretation of data, and in writing the manuscript. ILSI Europe facilitated scientific meetings and coordinated the overall project management and administrative tasks relating to the completion of this work. The research reported is the result of a scientific evaluation in line with ILSI Europe's framework to provide a pre-competitive setting for public-private partnership (PPP). For further information about ILSI Europe, please email info@ilsieurope.be or call + 32277100 14. The opinions expressed herein and the conclusions of this publication are those of the authors and do not represent the views of ILSI Europe nor those of its member companies.

\section{References}

1. Colombo A P V , Tanner A C R. The Role of Bacterial Biofilms in Dental Caries and Periodontal and Periimplant Diseases: A Historical Perspective. J Dent Res 2019; 98: 373-385.

2. Teles R, Sakellari D, Teles F et al. Relationships among gingival crevicular fluid biomarkers, clinical parameters of periodontal disease, and the subgingival microbiota. J Periodontol 2010; 81: 89-98.

3. Gao X, Jiang S, Koh D, Hsu C-Y S. Salivary biomarkers for dental caries. Periodontol 2000 2016; 70: 128-141.

4. Zaura E, Brandt B W, Prodan A et al. On the ecosystemic network of saliva in healthy young adults. ISME J 2017; 11: 1218-1231.

5. Belibasakis $\mathrm{G}$ N. Microbiological changes of the ageing oral cavity. Arch Oral Biol 2018: 96: 230-232.

6. Ebersole J L, Dawson 3rd D A, Huja P E et al. Age and periodontal health - immunological view. Curr Oral Health Rep 2018; 5: 229-241.

7. Van Dyke T E, van Winkelhoff A J. Infection and inflammatory mechanisms. J Periodontol 2013; 84(4 Suppl): S1-S7.

8. Lloyd-Price J, Abu-Ali G, Huttenhower C. The healthy human microbiome. Genome Med 2016; 8: 51

9. Marsh P D. Microbial ecology of dental plaque and its significance in health and disease. Adv Dent Res 1994; 8: 263-271.

10. Marsh P D. In Sickness and in Health - What Does the Oral Microbiome Mean to Us? An Ecological Perspective. Adv Dent Res 2018; 29: 60-65.

11. Rosier B T, De Jager M, Zaura E, Krom B P. Historical and contemporary hypotheses on the development of oral diseases: are we there yet? Front Cell Infect Microbiol 2014; 4: 92.

12. Takahashi N, Nyvad B. The role of bacteria in the caries process: ecological perspectives. J Dent Res 2011; 90 : 294-303.

13. Eriksson L, Holgerson P L, Esberg A, Johansson I. Microbial Complexes and Caries in 17-Year-Olds with and without Streptococcus mutans. J Dent Res 2018; DOI: $10.1177 / 0022034517731758$.

14. Richards V P, Alvarez A J, Luce A R et al. Microbiomes of site-specific dental plaques from children with different caries status. Infect Immun 2017; DOI: 10.1128/ IAl.00106-17. 
15. Xiao J, Klein M I, Falsetta M L et al. The exopolysaccharide matrix modulates the interaction between $3 \mathrm{D}$ architecture and virulence of a mixedspecies oral biofilm. PLoS Pathog 2012; DOI: 10.1371/ journal.ppat.1002623.

16. Bowen W H, Burne RA, Wu H, Koo H Oral Biofilms: Pathogens, Matrix, and Polymicrobial Interactions in Microenvironments. Trends Microbiol 2018; 26: 229-242.

17. Socransky SS, Haffajee A D, Cugini M A, Smith C, Kent Jr R L. Microbial complexes in subgingival plaque. J Clin Periodontol 1998; 25: 134-144.

18. Aruni A W, Mishra A, Dou Y, Chioma O, Hamilton B N Fletcher $\mathrm{H}$ M. Filifactor alocis - a new emerging periodontal pathogen. Microbes Infect 2015; 17: 517-530.

19. Hajishengallis $G$, Liang $S$, Payne M A et al. Low-abundance biofilm species orchestrates inflammatory periodontal disease through the commensal microbiota and complement. Cell Host Microbe 2011; 10: 497-506.

20. Hajishengallis $G$, Lamont R J. Dancing with the stars: How choreographed bacterial interactions dictate nososymbiocity and give rise to keystone pathogens, accessory pathogens, and pathobionts. Trends Microbiol 2016; 24: 477-489.

21. Chapple I L, Bouchard P, Cagetti M G et al. Interaction of lifestyle, behaviour or systemic diseases with dental caries and periodontal diseases: consensus report of group 2 of the joint EFP/ORCA workshop on the boundaries between caries and periodontal diseases. J Clin Periodontol 2017; 44 Suppl 18: S39-S51.

22. GBD 2017 Oral Disorders Collaborators; Bernabe $E_{\text {, }}$ Marcenes W, Hernandez C R et al. Global, Regional, and National Levels and Trends in Burden of Oral Conditions from 1990 to 2017: A Systematic Analysis for the Global Burden of Disease 2017 Study. J Dent Res 2020; 99: 362-373.

23. Hajishengallis E, Parsaei Y, Klein M I, Koo H. Advances in the microbial aetiology and pathogenesis of early childhood caries. Mol Oral Microbiol 2017; 32: 24-34.

24. Gavriilidou N N, Belibasakis $G$ N, Root caries: the intersection between periodontal disease and dental caries in the course of ageing. Br Dent J 2019; 227: 1063-1067.

25. Armitage G C, Cullinan M P. Comparison of the clinical features of chronic and aggressive periodontitis. Periodontol 2000 2010; 53: 12-27.

26. Moynihan P, Tanner L M, Holmes R D et al. Systematic Review of Evidence Pertaining to Factors That Modify Risk of Early Childhood Caries. JDR Clin Trans Res 2019; 4: 202-216.

27. Feres M, Teles F, Teles R, Figueiredo L C, Faveri M. The subgingival periodontal microbiota of the aging mouth. Periodontol 2000 2016: 72: 30-53.

28. Caporaso J G, Lauber C L, Costello E K et al. Moving pictures of the human microbiome. Genome Biol 2011; 12: R50.

29. Relman D A. The human microbiome: ecosystem resilience and health. Nutr Rev 2012; 70 Suppl 1: S2-S9.

30. Rosier B T. Marsh P D, Mira A. Resilience of the Oral Microbiota in Health: Mechanisms That Prevent Dysbiosis. J Dent Res 2018; 97: 371-380.

31. Hajishengallis G, Lamont R J, Graves D T. The enduring importance of animal models in understanding periodontal disease. Virulence 2015; 6: 229-235.
32. Baraniya D, Naginyte M, Chen T et al. Modeling Normal and Dysbiotic Subgingival Microbiomes: Effect of Nutrients. J Dent Res 2020: 99: 695-702.

33. Wu YY, Westwater C, Xiao E, Corrêa J D, Xiao W M, Graves D T. Establishment of oral bacterial communities in germ-free mice and the influence of recipient age. Mol Oral Microbiol 2018; 33: 38-46.

34. Sandal I, Karydis A, Luo J et al. Bone loss and aggravated autoimmune arthritis in HLA-DRbeta1-bearing humanized mice following oral challenge with Porphyromonas gingivalis. Arthritis Res Ther 2016; 18: 249.

35. Zemouri C, Jakubovics N S, Crielaard W et al. Resistance and resilience to experimental gingivitis: a systematic scoping review. BMC Oral Health 2019; 19: 212.

36. Shi B, Chang M, Martin J et al. Dynamic changes in the subgingival microbiome and their potential for diagnosis and prognosis of periodontitis. mBio 2015; DOI: 10.1128/mBio.01926-14.

37. Liu G, Luan Q, Chen F, Chen Z, Zhang Q, Yu X. Shift in the subgingival microbiome following scaling and root planing in generalized aggressive periodontitis. J Clin Periodontol 2018; 45: 440-452.

38. Darveau R P. Periodontitis: a polymicrobial disruption of host homeostasis. Nat Rev Microbiol 2010; 8: 481-490.

39. Cosseau C, Devine D A, Dullaghan E et al. The commensal Streptococcus salivarius K12 downregulates the innate immune responses of human epithelial cells and promotes host-microbe homeostasis. Infect Immun 2008; 76: 4163-4175.

40. Johansson I, Witkowska E, Kaveh B, Holgerson P L, Tanner A C R. The microbiome in populations with a low and high prevalence of caries. J Dent Res 2016; 95: 80-86.

41. Hardie J M, Thomson P L, South R J et al. A longitudinal epidemiological study on dental plaque and the development of dental caries-interim results after two years. J Dent Res 1977; DOI: 10.1177/00220345770560032401.

42. Loesche W J, Straffon L H. Longitudinal investigation of the role of Streptococcus mutans in human fissure decay. Infect Immun 1979; 26: 498-507.

43. Lang N P, Hotz P R, Gusberti F A, Joss A. Longitudinal clinical and microbiological study on the relationship between infection with Streptococcus mutans and the development of caries in humans. Oral Microbiol Immunol 1987; 2: 39-47.

44. Holgerson P L, Öhman C, Rönnlund A, Johansson I. Maturation of oral microbiota in children with or without dental caries. PLoS One 2015; DOI: 10.1371/ journal.pone.0128534.

45. Dzidic M, Collado M C, Abrahamsson T et al. Oral microbiome development during childhood: an ecological succession influenced by postnatal factors and associated with tooth decay. ISME J 2018; 12: 2292-2306.

46. Xu H, Tian J, Hao W et al. Oral Microbiome Shifts From Caries-Free to Caries-Affected Status in 3-Year-Old Chinese Children: A Longitudinal Study. Front Microbiol 2018; DOI: 10.3389/fmicb.2018.02009

47. Theilade E, Wright W H, Jensen S B, Löe H. Experimental gingivitis in man. II. A longitudinal clinical and bacteriological investigation. J Periodontal Res 1966; 1: 1-13.

48. Kilian M, Chapple I L C, Hannig M etal. The oral microbiome - an update for oral healthcare professionals. Br Dent J 2016; 221: 657-666.
49. Succurro A, Ebenhöh 0 . Review and perspective on mathematical modeling of microbial ecosystems. Biochem Soc Trans 2018; 46: 403-412.

50. Kreft J-U, Plugge C M, Prats C, Leveau J H J, Zhang W, Hellweger F L. From Genes to Ecosystems in Microbiology: Modeling Approaches and the Importance of Individuality. Front Microbiol 2017; DOI: 10.3389/fmicb.2017.02299.

51. Dibdin G H. and G. Reece, Computer-simulation of diffusion with reaction in dental plaque. Caries Res 1984; 18: 191-192.

52. Ilie O, van Loosdrecht M C M, Picioreanu C. Mathematical modelling of tooth demineralisation and $\mathrm{pH}$ profiles in dental plaque. J Theor Biol 2012; 309: 159-175.

53. Dibdin G H. Computer modelling of dental plaque in relation to dental caries. Microbial Ecol Health Dis 1990; DOI: 10.3109/08910609009140249.

54. Head D, Devine D A, Marsh P D. In silico modelling to differentiate the contribution of sugar frequency versus total amount in driving biofilm dysbiosis in dental caries. Sci Rep 2017; 7: 17413.

55. Papantonopoulos G, Takahashi K, Bountis T, Loos B G. Using cellular automata experiments to model periodontitis. Int J Bifurc Chaos 2013; DOI: 10.1142/ S0218127413500569.

56. Papantonopoulos G, Takahashi K, Bountis T, Loos B G Mathematical Modeling Suggests That Periodontitis Behaves as a Non-Linear Chaotic Dynamical Process. J Periodontol 2013; DOI: 10.1902/jop.2013.120637.

57. Song H-S, Cannon W R, Beliaev A S, Konopka A Mathematical modeling of microbial community dynamics: A methodological review. Processes 2014; 2: 711-752.

58. Rath $H$, Feng D, Neuweilier I, Stumpp N S, Nackenhorst U, Stiesch M. Biofilm formation by the oral pioneer colonizer Streptococcus gordonii: an experimental and numerical study. FEMS Microbiol Ecol 2017; DOI: 10.1093/femsec/fix010.

59. Martin B, Tamanai-Shacoori Z, Bronsard J et al. A new mathematical model of bacterial interactions in twospecies oral biofilms. PLoS One 2017; DOI: 10.1371/ journal.pone. 0173153

60. Head D A, Marsh P, Devine D A. Non-Lethal Control of the Cariogenic Potential of an Agent-Based Model for Dental Plaque. PLoS One. 2014; D0I: 10.1371/journal. pone.0105012.

61. Papantonopoulos G, Takahashi K, Bountis T, Loos B G. Artificial Neural Networks for the Diagnosis of Aggressive Periodontitis Trained by Immunologic Parameters. PLoS One 2014; DOI: 10.1371/journal. pone.0089757.

62. Topçuoğlu B D, Lesniak N A, Ruffin 4th M T, Wiens J, Schloss P D. A Framework for Effective Application of Machine Learning to Microbiome-Based Classification Problems. mBio 2020; DOI: 10.1128/ mBio.00434-20.

63. Seneviratne C J, Balan P, Suriyanarayanan et al. Oral microbiome-systemic link studies: perspectives on current limitations and future artificial intelligencebased approaches. Crit Rev Microbiol 2020; DOI: 10.1080/1040841X.2020.1766414.

64. Caton J G, Armitage G, Berglundh T etal. A new classification scheme for periodontal and peri-implant diseases and conditions - Introduction and key changes from the 1999 classification. J Periodontol 2018; 89 Suppl 1: $\$ 1-\$ 8$. 